\title{
An improved iCLIP protocol
}

Flora C. Y. Lee ${ }^{1,2}$, Anob M. Chakrabarti1 ${ }^{1}$, Heike Hänel ${ }^{3,{ }^{*},}$, Elisa Monzón-Casanova ${ }^{4,{ }^{*}}$, Martina Hallegger ${ }^{1,2}$, Cristina Militti ${ }^{1,2}$, Federica Capraro ${ }^{1,2}$, Christoph Sadée ${ }^{1}$, Patrick Toolan-Kerr ${ }^{1,2}$, Oscar Wilkins $^{1,2}$, Martin Turner ${ }^{4}$, Julian König ${ }^{3}$, Christopher R. Sibley5,6,, Jernej Ule ${ }^{1,2,8}$

1 The Francis Crick Institute, 1 Midland Road, London, NW1 1AT, UK

2UCL Queen Square Institute of Neurology, Department of Neuromuscular Diseases, University College London, London, WC1N 3BG, UK

3Institute of Molecular Biology (IMB), Ackermannweg 4, 55128 Mainz, Germany

${ }^{4}$ Immunology Programme, The Babraham Institute, Babraham Research Campus, Cambridge, CB22 3AT 5Institute of Quantitative Biology, Biochemistry and Biotechnology, School of Biological Sciences, Edinburgh University, 1 George Square, Edinburgh, EH8 9JZ, UK ${ }^{6}$ Simons Initiative for the Developing Brain, University of Edinburgh, Hugh Robson Building, George Square, Edinburgh, EH8 9XD, UK

${ }^{7}$ Department of Medicine, Division of Brain Sciences, Imperial College London, Burlington Danes, London, UK.

${ }^{8}$ National Institute of Chemistry, Hajdrihova ulica 19, 1000 Ljubljana, Slovenia

${ }^{*}$ These authors contributed equally

\section{Abstract}

Crosslinking and Immunoprecipitation (CLIP) is a powerful technique to obtain transcriptome-wide maps of in vivo protein-RNA interactions, which are important to understand the post-transcriptional mechanisms mediated by RNA binding proteins (RBPs). Many variant CLIP protocols have been developed to improve the efficiency and convenience of cDNA library preparation. Here we describe an improved individual nucleotide resolution CLIP protocol (iiCLIP), which can be completed within 4 days from UV crosslinking to libraries for sequencing. For benchmarking, we directly compared PTBP1 iiCLIP libraries with the iCLIP2 protocol produced under standardised conditions, and with public eCLIP and iCLIP PTBP1 data. We visualised enriched motifs surrounding the identified crosslink positions and RNA maps of these crosslinks around the alternative exons regulated by PTBP1. Notably, motif enrichment was higher in iiCLIP and iCLIP2 in comparison to public eCLIP and iCLIP, and we show how this impacts the specificity of RNA maps. In conclusion, iiCLIP is technically convenient and efficient, and enables production of highly specific datasets for identifying RBP binding sites. 


\section{Introduction}

Many techniques employing high-throughput sequencing are available for 'protein-centric' transcriptomic studies of protein-RNA interactions, including RIP, CLIP, TRIBE, APEX-seq and others (Hafner et al. 2021). Each technique has distinct advantages as well as limitations; some define the precise position of direct protein-RNA contacts, whereas others have lower resolution but also detect RNAs that are in spatial proximity (Ramanathan, Porter, and Khavari 2019). CLIP and its derived methods fall into the first category, requiring 'zero-distance' covalent crosslinking of in vivo proteinRNA contacts, usually mediated by UVC irradiation, to enable stringent purification of the RBP-ofinterest with their bound RNA (Ule et al. 2003; Lee and Ule 2018). This is coupled with library preparation and sequencing of the preserved RNA fragments.

Current variants of CLIP protocols can provide precise positional and quantitative information on the crosslink events, which is important in deciphering the regulatory functions of RBPs. These protocols vary in sensitivity and specificity due to differences in the stringency of purification to avoid reassociations during purification of protein-RNA complex, enzymatic biases, and efficiency of cDNA library preparation protocol, among others (Lee and Ule 2018). Many current CLIP variants identify crosslink positions by the analysis of cDNA truncations as established in iCLIP (König et al. 2010). Compared to the original CLIP protocol (Ule et al. 2005), iCLIP experimentally differs mainly at the adapter ligation steps. The original CLIP protocol ligated two adapters (SeqRv and SeqFw, (Lee and Ule 2018)) to the isolated RNA fragments at the 3' and 5' ends, respectively, whereas in iCLIP the SeqFw adapter sequence is introduced to the cDNAs during reverse transcription (RT), which establishes a way to amplify truncated cDNAs. The SeqFw adapter sequence, experimental barcode and UMls are introduced as part of the RT primer. By amplifying the truncated cDNAs and adding the use of UMIs, iCLIP increased the coverage, nucleotide resolution and quantitative nature of the method. Since then, multiple additional 'truncation-based' CLIP protocols were published (Lee and Ule 2018; Van Nostrand et al. 2016; Zarnegar et al. 2016; Buchbender et al. 2019), each with its own further optimisations. This led to dozens of available variant CLIP protocols, and thus it can be challenging to select a suitable variant. Here, we present an improved iCLIP protocol, referred to as 'iiCLIP', where we combined several published and new optimisations to increase its sensitivity and convenience.

\section{Development of iiCLIP}

We recently employed the iiCLIP protocol for the comparative studies of RBP binding upon changes in condensation. The reproducibility of this streamlined approach allowed us to compare across a 
panel of TDP-43 C-terminal mutant variants, and thereby derive principles for condensationdependent assembly on RNA-binding regions which have unusually long stretches of dispersed UGmotifs (Hallegger et al. 2021). The rationale of the improvements compared to iCLIP are explained in Table 1; the schematic overview of the full protocol is found in Figure 1. Notably, iiCLIP offers an alternative to radioactive labelling of RNPs, which was first introduced by irCLIP for quality control at the SDS-PAGE step (Zarnegar et al. 2016), while retaining similar enzymatic steps of iCLIP but with improved efficiency, combined with Ampure beads-based purifications to streamline the library preparation procedure and minimise loss of material. Thus, the resulting data from iiCLIP experiments is expected to have similar or better resolution and specificity compared to iCLIP, with greater efficiency in library preparation, and we confirmed this in the benchmarking comparisons presented below (Figures 2-4).

\section{Benchmarking of iiCLIP: a controlled comparison using the same input material}

To assess the performance of iiCLIP, we performed several comparisons. First, to test that iiCLIP can be easily adopted by additional labs, we designed a comparison between CLIP protocol developer and non-developer labs. Two iiCLIP libraries were generated in a non-developer lab, and a single iiCLIP library was produced in the iiCLIP developer lab for the purpose of this study (Supplementary Table 1). Second, we directly benchmarked iiCLIP against the recently published iCLIP2 protocol (Buchbender et al. 2019). iCLIP2 was shown to increase the sensitivity of iCLIP by an order of magnitude and can be completed within four days (Buchbender et al. 2019). Like iCLIP, iCLIP2 contains the radioactive labeling step to monitor optimal conditions of immunoprecipitation and RNA fragmentation. The iiCLIP protocol differs from iCLIP2 in the non-radioactive visualisation of the protein-RNA complex, conditions of RNA ligation, cDNA ligation and in the post-RT conditions of cDNA purification and amplification.

These comparisons were controlled for using the same input cells, antibodies and RNase treatment. A batch of cells was prepared and UVC crosslinked in the Ule lab, which were sent along with a tested batch of RNase and antibody to two other laboratories. Thus, the experiments were performed with identical RNase treatment and immunoprecipitation conditions. After sequencing using the described conditions (Table S1), reads were processed through the primary analysis pipeline on the iMaps web server (imaps.genialis.com/) to identify crosslink positions by mapping cDNA truncations and to quantify cDNA counts per position using UMIs, which applies for all protocols compared in this study (Figure2A, Tables S2 and S3). 


\section{Validation of sensitivity and specificity of data through PTBP1 motif analysis}

The CLIP method has been developed with the purpose of obtaining data with high specificity for the direct RNA binding sites of an RBP. An additional aim during iiCLIP development has been to maximise data sensitivity by increasing the complexity of the produced cDNA library, and thus its capacity to comprehensively identify binding sites across a wider dynamic range of RNA expression and with less input material. However, it is important that changes in data specificity are carefully assessed whenever changes are made that aim at higher sensitivity (Hafner et al. 2021). Here, we wished to gain a comparative perspective on both the sensitivity and specificity of data obtained by both protocols, which we produce in this study, and with representative public data from other published CLIP variants. We focused on PTBP1, a well-characterised nuclear RBP and splicing regulator that has been used by several labs to benchmark their own CLIP variants, which has already enabled some meta-analyses based on these published data (Chakrabarti et al. 2018; Haberman et al. 2017; Van Nostrand et al. 2016; Zarnegar et al. 2016; Gu et al. 2018). Specificity of PTBP1 CLIP data can be assessed through sequence motif enrichment around crosslinks, as its binding to UC-rich RNA motifs has been well characterised with in vitro biochemistry and structural studies, and is supported by evidence of motif enrichment around regulated exons (Oberstrass et al. 2005; Spellman et al. 2005; Chou et al. 2000; Cereda et al. 2014). Sensitivity for functionally important sites can be shown with an RNA map (Ule et al. 2006), which demonstrates how PTBP1 binding around regulated exons has bidirectional outcomes on RNA processing in a positiondependent manner (Witten and Ule 2011; Xue et al. 2009; Llorian et al. 2010). Therefore the analysis of PTBP1 CLIP data can provide information on sensitivity and specificity of the crosslink positions and binding peaks through multiple means (Chakrabarti et al. 2018), which we have exploited here for comparative analysis of CLIP variants.

We produced iCLIP2 and iiCLIP data of PTBP1 immunoprecipitation from 1 million HEK293 cells for the purpose of controlled comparison. For the purpose of nomenclature, we refer to data produced by developer labs as iCLIP2_d and iiCLIP_d, whereas the data produced in non-developer lab is referred to as iiCLIP_nd. To contextualise these new data with existing protocols, we reprocessed public iCLIP and ENCODE eCLIP raw data on PTBP1 with the identical computational pipeline on iMaps (Table S3). First, we examined the regional distribution of sequenced cDNAs (Figure2B, C). As expected, iCLIP2 and iiCLIP PTBP1 cDNAs predominantly map to introns, similar to public iCLIP (Figure 2B, C). Interestingly, we observed differences in the proportion of reads mapping to intergenic and noncoding RNAs, which is highest in iiCLIP_d1 (18\% and 12\%) and lowest in public iCLIP (3\% and 8\%) (Figure 2B). On the other hand, public eCLIP data has a $\sim 10$-fold greater proportion of CDS reads ( $11 \%$ in eCLIP compared to $0.3-1.2 \%$ in other variant protocols). Therefore, whilst the regional 
distribution of sequenced reads can be protocol- and experiment- dependent, iCLIP2, iiCLIP and public iCLIP are generally similar when comparing cDNAs which map to mRNA regions (Figure $2 \mathrm{C}$ ).

Next, we assessed the occurrence of PTBP1-binding motifs around the intronic crosslink positions. As is standard for iCLIP and eCLIP, crosslink sites were assigned to the genomic coordinate preceding the start of CDNA inserts. We then evaluated the coverage around crosslink positions of $12 \mathrm{UC}$-rich pentamers that were found to be most enriched in previous PTBP1 CLIP studies (Haberman et al. 2017). These pentamers were highly enriched at the crosslink positions of all datasets evaluated here, but with major differences in the extent of enrichment in the region within 10nt of crosslink sites, from the minimum of $18.7 \%$ (in eCLIP) to the maximum of $37.9 \%$ (in iiCLIP_d) (Figures 2D and S1E ). We were surprised to find that the iiCLIP_nd had lower enrichment than iiCLIP_d, such that iiCLIP_nd was similar to the public iCLIP (19.3-20.6\%). Reassuringly, motif enrichment around 3'UTR, intergenic and ncRNA crosslink positions showed similar variability across the libraries as in introns, and the strong occurence of PTBP1 motifs generally supported the specificity of crosslink sites identified in all of these regions (Figure S1A-C, E). However, eCLIP crosslinks in CDS did not show motif enrichment compared with SMInput data, suggesting that the increased abundance of CDS cDNAs may derive from background contamination (Figure S1D). Of additional interest, the highest motif enrichment of all regions was seen in the intergenic RNA crosslinking sites (Figure S1A), which were most represented in iiCLIP_d, highlighting the need to better understand the sources and functionality of these sites (Agostini et al. 2021).

Notably, we also observed major variations in the broader profiles of motif enrichment around crosslink sites. In eCLIP, motif enrichment was higher upstream than downstream of crosslink sites, while in all other datasets the enrichment was higher downstream of crosslink sites (Figures 2C and $\mathrm{S1})$. We believe this is likely the result of higher crosslinking energy used in eCLIP $\left(400 \mathrm{~mJ} / \mathrm{cm}^{2}\right)$, as compared with all other variants $\left(150 \mathrm{~mJ} / \mathrm{cm}^{2}\right)$ (Van Nostrand et al. 2016; Huppertz et al. 2014). Namely, high crosslinking increases the chance for multiple PTBP1 crosslink events on binding regions that contain a sequence of multiple motifs, and as a result, cDNAs will truncate closer to the 3'end of the bound RNA regions. Moreover, the iiCLIP_d and iCLIP2 datasets have the broadest enrichment of motifs. Specifically, in the region 11-50nt downstream of crosslink sites, average motif enrichment is $14.3 \%$ (eCLIP), 17.5-19.5\% (iCLIP and iiCLIP_nd), $23.7 \%$ (iCLIP2) and 36.5\% (iiCLIP_d). It has been shown that such broad patterns of enrichment can be derived from highly multivalent, long binding regions, which are more challenging to map in the genome due to their repetitive nature, and therefore long cDNAs are required to detect crosslink sites in these regions 
(Haberman et al. 2017). Hence, the shorter read length of cDNAs in iiCLIP_nd1 may contribute partly to differences in the ability to identify crosslinks in the highly multivalent UC-rich regions (Figure 2E).

\section{Examination of data specificity and sensitivity by RNA maps analysis}

As a further independent approach to assess the specificity and sensitivity of community CLIP experiments, we examined their capacity to identify in vivo RNA binding that is relevant to the position-dependent regulatory role played by PTBP1 in alternative splicing. For this, we analysed RNA maps of PTBP1 for the different CLIP variants, which examines RBP binding sites around the exons that are differentially spliced upon depletion of the RBP (Figures 3 and S2) (Ule et al. 2006). We used exons which were identified either as silenced or enhanced by PTBP1 from RNA-seq analysis of HEK293 cells after PTBP1/2 knockdown (Gueroussov et al. 2015) (Figures S2A,3), microarray analysis upon PTBP1/PTBP2 knockdown in HeLa cells (Llorian et al. 2010) (Figure S2B), or from ENCODE RNA-seq analysis of K562 cells after PTBP1-targeted CRISPR treatment (Van Nostrand et al. 2020) (Figure S2C). We analysed the coverage of crosslink events up to 50nt into the exonic and 300nt into the intronic sequence flanking the splice sites of the affected exons. The strongest enrichment of PTBP1 crosslink events was seen in the upstream -100...0 window of the 3' splice site of PTBP1-silenced exons, as compared to control exons (Figure S2A-C). This enrichment could be observed regardless of whether the PTBP1 regulated exons were defined from analysis of cell-line matched datasets (HEK293 for iiCLIP and iCLIP2 libraries, HeLa for public iCLIP and K562 for public eCLIP data).

To further understand how different datasets perform in detecting the proximal 3' splice site enrichment pattern around silenced exons, we classified silenced exons from the HEK293 RNA-seq based on the relative coverage of crosslinks from the iiCLIP_d1 and iiCLIP_nd1 dataset in the 100nt window upstream of the 3' splice site and analysed the RNA maps crosslink metaprofiles for these classes separately as a measure for sensitivity (Figure $3 A$ ). Silenced exons which were detected preferentially in iiCLIP_d1 had slightly broader (up to -100 relative to 3' splice site) and higher PTBP1 motif coverage compared to those exons with relatively greater crosslink coverage in iiCLIP_nd1 (Figure 3B\&C), which likely reflects the differences in motif coverage around crosslink events in the two datasets (Figure 2C). Notably, a third class of PTBP1-silenced exons that had no detectable crosslinks in both datasets showed a lower enrichment of motifs restricted to a sharp peak directly upstream of the 3' splice site, a pattern of motif coverage more similar to the control exons (Figure 3D\&E). The difference in crosslinking coverage for the three classes of silenced exons is reproduced across all PTBP1 datasets (Figure $3 \mathrm{~A}$ ). Thus all CLIP variants preferentially identified silenced exons 
harbouring multivalent RNA-binding regions upstream of the 3' splice site, which are candidate exons for direct PTBP1 repression.

Finally, we compared the PTBP1 crosslinking profiles identified by CLIP variants at individual RNAs. All iiCLIP and iCLIP2 libraries produced for this study show a highly reproducible distribution of crosslink positions and frequency on the ncRNAs XIST and MALAT1 (Figure 4A). Compared to the input control produced in parallel to iiCLIP (iiclip_d_input, see methods), all of these PTBP1 libraries show hundred-fold enrichment of signal in the $E$ repeat region of Xist, whereas the normalised signal across almost all positions of MALAT1 was lower in iiCLIP compared to input control, in agreement with the known functional binding of PTBP1 to Xist (Pandya-Jones et al. 2020) but not MALAT1. We next analysed the RNAs containing PTBP1-repressed exons, where we also observed highly reproducible crosslink signal across all the CLIP libraries produced in this study (Figure 4B). Thus, by performing comparative CLIP analysis across multiple labs using controlled batches of input material and immunoprecipitation conditions, we show that both protocols are capable of generating highly specific data with similar sensitivity in spite of their major downstream differences in the conditions of reverse transcription, cDNA ligation enzymes, strategies of cDNA purification and PCR amplification. In summary, the iiCLIP protocol can be set up and completed effectively in a non-developer lab, and overall shows similar sensitivity and specificity to the latest variant CLIP protocols.

\section{Discussion}

\section{Sources of biological and technical background in CLIP}

A critical aspect of CLIP data analysis is to derive confident binding sites from the crosslink events detected in the experiment, by identifying positions with signal above the background distribution (Chakrabarti et al. 2018). There are two sources of background to be considered: intrinsic and extrinsic. Biological noise in CLIP data can arise from true RBP crosslinking capturing stochastic RBP-RNA interactions which occur in addition to binding at cognate high-affinity sites, which can be referred to as 'intrinsic background' (Hafner et al. 2021). Extrinsic background, however, partly derives from RNA fragments crosslinked to co-purified RBPs. Such background can be relevant for understanding the function of the RBP-of-interest in case the co-purified RBPs are interacting partners within larger RNP complexes, though it may obscure the intrinsic specificity of the RBP. Another type of extrinsic background derives from sources which do not biologically relate to the RBPof-interest, for example contamination with abundant RNA fragments or abundant cellular RBPs with their crosslinked RNAs (Hafner et al. 2021). This is most likely to be cell-state and protocoldependent. In addition to these sources of background, CLIP data are affected by technical biases in the library preparation protocol. These dynamically alter the distribution of final sequenced reads such 
that their observed abundance in a CLIP experiment may not faithfully represent the repertoire of isolated RNA fragments.

One approach to assess the extrinsic background of CLIP is to sequence input libraries, as done routinely for eCLIP SMInput samples that loads the crosslinked lysate on the gel and isolates RNA from a size-matched region of the membrane followed by in-solution dephosphorylation and adapter ligation. Here we used a modified approach to producing input data, where crosslinked protein-RNA complexes are captured first on beads (Moggridge et al. 2018), allowing parallel processing of both IP and input samples and on-bead dephosphorylation and adapter ligation, which makes the conditions of the input sample fully comparable to the IP sample in the iiCLIP protocol. We procured iiCLIP_d_input in conjunction with the PTBP1 iiCLIP_d1. Similar to the eCLIP SMInput, the iiCLIP_d_input sample also shows some enrichment in CDS exons (Figures S2A, 1B). However, we observed negligible overlap in the signal between the PTBP1 iiCLIP_d and corresponding input libraries, both at the metaprofile RNA splicing map level and individual binding site level (Figures 3, 4, S2). While the CDS enrichment from PTBP1 eCLIP warrants background normalisation by eCLIP SMInput, there is no CDS crosslink enrichment in PTBP1 iiCLIP and thus input normalisation is not required to remove such signal. Instead, peak-calling approaches that model the intrinsic background from iiCLIP data itself might be more appropriate for iiCLIP, and input data could instead serve as a proxy to account for RNA abundance (Hafner et al. 2021).

\section{Limitations of iiCLIP protocol and future directions}

We showed iiCLIP has good sensitivity for 1 million cells, however when input material is low, adapter-primer and RT primer concatemer artefacts do emerge during PCR in iiCLIP libraries. This indicates that whilst we have introduced enzymatic removal steps after ligation and reverse transcription, they are insufficient to remove all excess oligos. In cases of visible artefact contamination, these artefacts should be removed by gel purification after PCR amplification, which minimises the loss of unique cDNAs, prior to sequencing. Further optimisations on adapter and RT primer sequences and/or annealing temperatures might minimise these artefacts.

Moreover, iiCLIP shares similar limitations with other CLIP variant protocols (Lee and Ule 2018). The CLIP approach relies on a specific antibody. If this is not available, it may be possible to express epitope-tagged proteins in the relevant cells lines or other types of input material. Orthogonal methods to CLIP may also be applicable, such as ones which rely on mapping of RNA-tagging or editing events (Lapointe et al. 2015; McMahon et al. 2016; Brannan et al. 2021). These are especially valuable if the protein of interest does not crosslink well, for example when indirect protein-RNA 
interactions are of interest to study functions of a protein-complex. CLIP can also be used comparatively to understand selective remodelling of RNP networks in vivo (Hallegger et al. 2021), and to take full advantage of such analyses, further development of experimental and computational approaches will be valuable.

As CLIP protocols continue to evolve, it is important to assess both sensitivity and library specificity with computational strategies using a benchmarking RBP or ideally multiple RBPs with different binding characteristics. Accordingly, we advocate caution in the interpretation of improvements which are primarily based on measurements of library depth. Resolving the next experimental and computational challenges for CLIP technologies will allow us to design robust experiments with limited input material to probe dynamic biological contexts, thereby advancing our understanding of the principles underlying the assembly of protein-RNA complexes and their coordinated regulatory functions. 


\section{Author Contributions}

F.C.Y.L. and J.U. conceptualised the project. F.C.Y.L., M.H., C.M., F.C., C.Sa., P.T.-K., O.W., C.R.S. contributed to iiCLIP protocol optimisations. J.U. supervised iiCLIP development. F.C.Y.L. prepared CLIP materials for benchmarking. F.C.Y.L., H.H.,E.M.-C. performed CLIP experiments for benchmarking. A.M.C. provided software. A.M.C. and F.C.Y.L. performed CLIP benchmarking analysis. J.U. supervised CLIP analysis. M.T., J.K. and J.U. supervised CLIP benchmarking experiments. F.C.Y.L. and J.U. wrote the paper and protocol with input from all other authors.

\section{Acknowledgements}

The authors thank Charlotte Capitanchik for providing the HEK293 RNAseq alternative splicing analysis used for benchmarking and Klara Kuret for help on motif coverage; Jan Medenbach for providing the TS2126 RNA Ligase I (Circligase) expression plasmid; Ina Huppertz, Tom Schultz, Matthias Hentze, and the past and current members of Ule lab for valuable discussions and assistance. This research was funded in whole, or in part, by the Wellcome Trust [103760/Z/14/Z, 215593/Z/19/Z (to J.U.); 105202/Z/14/Z (to F.C.Y.L.)]. For the purpose of open access, the author has applied a CC BY public copyright licence to any Author Accepted Manuscript version arising from this submission. This research was funded additionally by the ERC [617837-Translate and 835300RNPdynamics (to J.U.)]; and Biotechnology and Biological Sciences Research Council (BBSRC) grants [BB/P01898X/1 and BBS/E/B/000C0428 (to E.M-C. and M.T.)]. The Francis Crick Institute receives its core funding from Cancer Research UK (FC001110), the UK Medical Research Council (FC001110), and the Wellcome Trust (FC001110).

Competing interests: C.R.S is inventor on a patent application covering specific elements of this method (i.e. adapter removal and size-matched input workflow). The other authors declare no competing interests. 


\section{Methods}

\section{Data availability}

Benchmarking datasets in this manuscript are described in Table S1 and S2. Sequencing data newly generated has been deposited to ArrayExpress (E-MTAB-10881). Processed CLIP data can be accessed on the iMaps webserver (https://imaps.goodwright.com/collections/1203/).

\section{Code availability}

Code used to generate all figures for iiCLIP benchmarking can be found on the github repository of this manuscript (https://github.com/ulelab/iiclip-benchmarking).

Shared conditions, cell pellets and reagents across benchmarking CLIP experiments against PTBP1 HEK293 cells were grown in monolayer to $90 \%$ confluency on $150 \mathrm{~mm}$ dishes with DMEM $10 \%$ FBS media prior to UVC crosslinking. Cells were washed once with $10 \mathrm{ml}$ of $1 \mathrm{x}$ PBS, and UVC crosslinked $(254 \mathrm{~nm})$ at $150 \mathrm{~mJ} / \mathrm{cm}^{2}$ on an ice-filled tray with a Stratalinker 2400 . Cells are then scraped off the dish on ice in PBS, and spun down at $250 \mathrm{G}$ at $4^{\circ} \mathrm{C}$. Subsequently, cells are resuspended in $10 \mathrm{ml}$ of 1x PBS and counted with Countess automated cell counter (ThermoFisher). 1 million cells were distributed to individual eppendorf tubes, pelleted at $500 \mathrm{G}$ at $4{ }^{\circ} \mathrm{C}$, and snap frozen on dry ice to be stored at $-80^{\circ} \mathrm{C}$. For distribution, cell pellets and RNase I (EN0602, Thermofisher scientific) aliquots were sent on dry ice, whereas mouse monoclonal anti-PTBP1 antibody (sc-56701, santacruz) were sent on ice.

iCLIP2 and iiCLIP were performed by lysing each cell pellet with $1 \mathrm{ml}$ of iCLIP lysis buffer (shared step across all protocols) with the following RNase I dilutions for 3 minutes at $37^{\circ} \mathrm{C}: 0.1 \mathrm{U}$ for 1 million cells. For each immunoprecipitation, 2ug of mouse monoclonal antibody (sc-56701) was used with $100 \mathrm{ul}$ of protein $\mathrm{G}$ beads for $1 \mathrm{ml}$ of protein lysate.

\section{iCLIP2 experiment}

iCLIP2 was performed as described in (Buchbender et al. 2019), with the shared crosslinked cells, and RNA fragmentation and immunoprecipitation conditions specified above. Libraries were sequenced on Miseq for SR150 (Table S1)

\section{iiCLIP protocol}

A step-by-step protocol is available upon request by contacting flora.lee@crick.ac.uk or jernej.ule@crick.ac.uk. 
Immunoprecipitation and wash conditions are the same as previous ICLIP, as described in (Huppertz et al. 2014). For the specific conditions used in iiCLIP_nd and iiCLIP_d, refer to Table S2.

\section{SeqRv adapter ligation}

After IP, beads were washed twice in high salt buffer and twice in PNK wash buffer, beads were incubated at $37^{\circ} \mathrm{C}$ for 40 minutes for 3 'end dephosphorylation in the following reaction mix: $8 \mu l 5 x$ PNK pH 6.5 buffer, $1 \mu$ PNK (NEB M0201L), 0.5 $\mu$ l FastAP alkaline phosphatase (ThermoFisher,

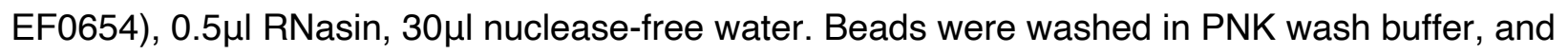
adapter ligation was performed on beads for overnight at $16^{\circ} \mathrm{C}$ or 75 minutes at $25^{\circ} \mathrm{C}$, with the following modified conditions: $6.3 \mu \mathrm{l}$ water, $3 \mu \mathrm{l}$ 10X ligation buffer (no DTT), $0.8 \mu \mathrm{l} 100 \%$ DMSO, $2.5 \mu \mathrm{l}$

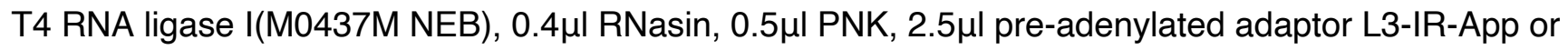
L3-XXX-App (stock $1 \mu \mathrm{M}), 9 \mu \mathrm{l}$ 50\% PEG8000.

\section{$\underline{\text { RecJ adapter removal }}$}

Beads were washed twice in high salt buffer and twice in PNK wash buffer after adapter ligation. The beads were then resuspended in the following reaction for adapter removal: $12 \mu$ l Nuclease-free water, $2 \mu \mathrm{l}$ NEB Buffer 2, 0.5 $\mu \mathrm{l}$ 5' Deadenylase (NEB M0331S), $1 \mu \mathrm{l} \mathrm{RecJ}$ endonuclease (NEB M0264S), $0.5 \mu \mathrm{l}$ RNasin, $4 \mu \mathrm{l} 50 \%$ PEG8000. The beads were incubated at $30^{\circ} \mathrm{C}$ for 1 hour, then 30 minutes at $37^{\circ} \mathrm{C}$ for 30 minutes.

\section{iCLIP SDS-PAGE and visualisation}

Beads were washed twice in high salt buffer and twice in PNK wash buffer after RecJ adapter removal. After washing, beads were then resuspended in $20 \mu \mathrm{l}$ of 1x NuPAGE loading buffer, and the RBP-RNA-linker complexes were eluted at $70^{\circ} \mathrm{C}$ for 1 minute. This is loaded in a NuPAGE $4-12 \%$ BisTris SDS-PAGE, run for 65 minutes at $180 \mathrm{~V}$ with MOPS buffer, and transferred to a nitrocellulose membrane for 2 hours at room temperature, 30V with 1x NuPAGE Transfer buffer with 10\% methanol. The membrane is then visualised at the LI-COR Odyssey-Clx. The pre-stained protein ladder is imaged at the 700nm channel whereas the RBP complexes ligated to the linker is visualised at the $800 \mathrm{~nm}$ channel (Zarnegar et al. 2016).

\section{RNA extraction}

The nitrocellulose membrane strips were digested with $10 \mu$ proteinase K (Roche, Ref no.: $03115828001)$ in $200 \mu$ l proteinase $\mathrm{K}$ SDS buffer (10mM Tris-HCl pH 7.4, 100mM NaCl, 1mM EDTA, $0.2 \%$ SDS) for 1 hour at $50 \mathrm{oC}$. The supernatant was transferred to a phase lock gel heavy tube 
(VWR, 713-2536). The RNA extracted were then phenol/chloroform extracted from the supernatant by adding 200 $\mu$ of Phenol:Chloroform:Isoamyl Alcohol 25:24:1 (Sigma, P3803), incubating shaking for 5 min at $30^{\circ} \mathrm{C}$, and centrifuged to separate the aqueous and organic phases. The aqueous phase was further extracted with $0.8 \mathrm{ml}$ of chloroform, centrifuged, and the upper phase containing the RNA is transferred to a new tube, and then precipitated with $20 \mu \mathrm{l} 3 \mathrm{M} \mathrm{NaAc}, 0.75 \mu \mathrm{l}$ glycoblue and $0.5 \mathrm{ml} \mathrm{EtOH}$ overnight at $-20^{\circ} \mathrm{C}$ (with or without addition of $0.5 \mu \mathrm{l} 1 \mu \mathrm{M}$ barcoded RT primers, Table S1). The RNA is pelleted for 30 minutes at $4^{\circ} \mathrm{C}$ at $13000 \mathrm{G}$.

\section{$\underline{\text { Reverse transcription and RT primer removal }}$}

The RNA is resuspended in 5.5 $\mu$ (SSIV reaction without PEG8000) or $5 \mu \mathrm{l}$ (for reaction with PEG8000) water then reverse transcribed with barcoded RT primers using the Superscript IV (SSIV) Kit with the following condition:

Add $0.5 \mu \mathrm{l} 1 \mu \mathrm{M}$ RT primer, $0.5 \mu \mathrm{l} 10 \mathrm{mM}$ dNTP, denature at $65^{\circ} \mathrm{C}$ for 5 minutes, ramp down to 25 . Hold at $25^{\circ} \mathrm{C}$ until addition of $2 \mu \mathrm{I} 5 \mathrm{x}$ SSIV buffer, $0.5 \mu \mathrm{l} 0.1 \mathrm{M}$ DTT, $0.25 \mu \mathrm{l}$ RNasin, $0.25 \mu \mathrm{ISSIV}$, and (see Table S1) $1 \mu \mathrm{l} 50 \%$ PEG8000. Then incubate in a thermocycler with the following program: $25^{\circ} \mathrm{C}, 5$ minutes. $50^{\circ} \mathrm{C}, 5$ minutes. $55^{\circ} \mathrm{C}, 5$ minutes. $4^{\circ} \mathrm{C}$, Hold.

After $\mathrm{RT}$, the reaction incubated with Exonuclease I $(2 \mathrm{U}$, add $1 \mu \mathrm{l}$ of a 10 -fold dilution of NEB Exonuclease I) for 15 minutes at $37^{\circ} \mathrm{C} .1 .5 \mu \mathrm{l} 0.5 \mathrm{M}$ EDTA is added to inactivate Exol, then RNA is degraded by alkaline hydrolysis at $85^{\circ} \mathrm{C}$ for 15 minutes by adding $1.25 \mu \mathrm{l} 1 \mathrm{M} \mathrm{NaOH}$. Afterwards, this is neutralised with $1.25 \mu \mathrm{l} 1 \mathrm{M} \mathrm{HCl}$.

\section{cDNA purification with AmPURE beads}

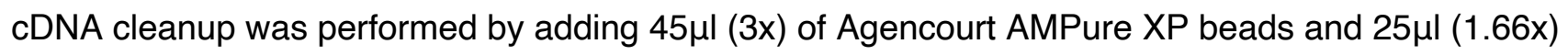
Isopropanol for capture for 5 minutes at room temperature, then magnetically separated. Supernatant was removed and beads were washed twice, each lasting 30 seconds, by overlaying the beads with $200 \mu \mathrm{l} 85 \% \mathrm{EtOH}$. After removal and evaporation of $\mathrm{EtOH}$, cDNA was eluted back in $9 \mu \mathrm{l}$ (followed by commercial circligase) or $6.75 \mu$ (followed by in-house TS2126) water.

cDNA circularisation

Eluted cDNA was moved to a new PCR tube and mixed with CircLigase II (Epicentre) reaction mix containing: $1.5 \mu \mathrm{l}$ 10x CircLigase II Buffer, $0.75 \mu$ CircLigase II, $0.75 \mu \mathrm{l} 50 \mathrm{mM} \mathrm{MnCl} 2,3 \mu \mathrm{l} 5 \mathrm{M}$ betaine, or mixed with in-house TS2126 reaction mix containing: $3 \mu \mathrm{l} 5 \mathrm{x}$ TS2126 buffer, $0.75 \mu \mathrm{l} 50 \mathrm{mM} \mathrm{MnCl}$, $0.75 \mu \mathrm{l} 1 \mathrm{mM}$ ATP, $0.75 \mu \mathrm{l}$ purified TS2126, $3 \mu \mathrm{l} 5 \mathrm{M}$ betaine. Circularisation reaction was incubated at 
$600 \mathrm{C}$ for 2 hours. Circularised cDNA is purified using the same AMPure XP beads procedure as before. cDNA is eluted in $10 \mu \mathrm{l}$ of water and can be stored at $-20^{\circ} \mathrm{C}$.

iiCLIP input experiment

$50 \mu \mathrm{l}$ of input crosslinked lysate was mixed with $50 \mu \mathrm{l}$ of SP3 beads, which were pre-washed and resuspended in water (Moggridge et al. 2018). $100 \mu \mathrm{l}$ of $100 \% \mathrm{EtOH}$ was mixed with the bead/lysate mixture and incubated for the duration of immunoprecipitation at $4 \mathrm{OC}$ for capture of protein-RNA complexes. The input sample then underwent the same iiCLIP procedure as the IP samples beginning from the wash steps before 3'end dephosphorylation. To assay the global input back in the iiCLIP experiment, RNA fragments were extracted from 40-250 kDa on the SDS-PAGE.

\section{CLIP analysis}

All CLIP sequencing data were uploaded to the iMaps webserver (imaps.genialis.com) for standardised primary analysis, for demultiplexing by experimental barcodes, UMI identification, adapter trimming, STAR pre-mapping to rRNAs and tRNAs, STAR alignment to genome, crosslink site assignment using read starts and duplicate removal by UMI sequence.

Figures were produced in Rstudio and code is available at (https://github.com/ulelab/iiclipbenchmarking).

\section{Public eCLIP and ICLIP PTBP1 data}

To compare iiCLIP libraries to public data, 2x K562 eCLIP replicates and 4x HeLa iCLIP replicates were merged using bedtools. Fastqs for eCLIP experiments (ENCFF689XJE, ENCFF273VIJ, ENCFF960RXN) were obtained from the ENCODE consortium project (https://www.encodeproject.org/).

\section{eCLIP pre-processing}

The reverse read (R2) was used for analysis as 5' end of R2 contains the UMI sequence followed by the site at which reverse transcription has truncated. To control for double adapter ligation events, eCLIP R2 fastq files were pre-processed according to the eCLIP SOP (Van Nostrand et al. 2016), with two rounds of cutadapt (performed on iMaps) equivalent to the following parameters: 1st round 
CCAAGATCGGAAGAG -a GTAGATCGGAAGAGC -a CAAGATCGGAAGAGC -a TAGATCGGAAGAGCG -a AAGATCGGAAGAGCG -a AGATCGGAAGAGCGT -a GATCGGAAGAGCGTC -a ATCGGAAGAGCGTCG -a TCGGAAGAGCGTCGT -a CGGAAGAGCGTCGTG -a GGAAGAGCGTCGTGT -m 18 --times 1 --match-read-wildcards --errorrate $0.1-01$

2nd round

-a AACTTGTAGATCGGA -a AGGACCAAGATCGGA -a ACTTGTAGATCGGAA -a GGACCAAGATCGGAA -a CTTGTAGATCGGAAG -a GACCAAGATCGGAAG -a TTGTAGATCGGAAGA -a ACCAAGATCGGAAGA -a TGTAGATCGGAAGAG -a CCAAGATCGGAAGAG -a GTAGATCGGAAGAGC -a CAAGATCGGAAGAGC -a TAGATCGGAAGAGCG -a AAGATCGGAAGAGCG -a AGATCGGAAGAGCGT -a GATCGGAAGAGCGTC -a ATCGGAAGAGCGTCG -a TCGGAAGAGCGTCGT -a CGGAAGAGCGTCGTG -a GGAAGAGCGTCGTGT -m 18 --times 1 --match-read-wildcards --errorrate $0.1-05$

Pre-processed fastq files were analysed by the primary analysis pipeline on iMaps without demultiplexing and adapter trimming.

\section{PTBP1 motif coverage}

PTBP1 enriched pentamers were previously identified in (Haberman et al. 2017). The top 12 pentamers ("TCTTT", "CTTTC", "TCTTC", "СTTCT", "TCTCT", "СTCTC", "TTTCT", "TTCTC", "TTCTT", "TTTTC", "TCCTT", "СTCTT") were selected for further analysis. Crosslinks weighted by cDNA count with score capping at the 95th percentile on a regional basis were subsampled to ten thousand (five thousand for CDS as this region contains less than ten thousand crosslinks in some libraries) without replacement for calculation of motif coverage around crosslink positions.

\section{PTBP1 RNA splicing map analysis}

Microarray data of PTBP1 KD was taken from (Llorian et al. 2010). Exons were defined using ASPIRE3 comparing KD to WT data to be control exons (IdIRankl <0.1), silenced by PTB (dIRank < 0.1) or activated by PTB (dIRank > - 0.1). K562 RNAseq data of PTBP1 was taken from ENCODE PTBP1 CRISPR RNA-seq (ENCSR415DJT) and control (ENCSR163JUC). HEK293 RNAseq data of PTBP1/2KD was obtained from a previous publication (Gueroussov et al. 2015). Skipped exons were detected using rMATS (Shen et al. 2014) using only junction counts for K562 and exon and junction counts for HEK293 with a p-value threshold of 0.05 and FDR threshold of 0.1. Silenced and enhanced exons were defined using an inclusion level difference threshold of 0.05 ; control exons were selected 
as those with a p-value greater than 0.1 , an FDR value greater than 0.1 , an mean inclusion level of less than 0.9, and an inclusion level difference less than 0.05. Because rMATS evaluates alternative splicing at the junction/event level, it is possible for the same exon to be duplicated within and across groups. Therefore duplicate entries within each group were collapsed; and exons which overlapped both silenced and enhanced were filtered from the analysis, as well as control exons which overlapped silenced or enhanced exons were removed from the control set. To plot the metaprofile, crosslinks weighted by cDNA counts were score-capped at the 99th percentile to minimise the contribution of outliers. To remove positions which are outliers in crosslinking cDNA counts due to the presence of other abundant RNA species (such as snoRNA), crosslinks which map to regions falling outside of 'CDS' and 'intron' were masked from the analysis, defined in a hierarchically segmented GTF annotation (based on gencode v27 hg38 annotation) supplied in iMaps computed by the iCount package (see https://icount.readthedocs.io/en/latest/_modules/iCount/genomes/segment.html?highlight=icount). Crosslinking coverage around splice sites were obtained with bedtools coverage function (Quinlan and Hall 2010).

\section{Motif maps}

Silenced exons from HEK293 PTBP1/2 KD RNAseq were separated into 3 classes based on crosslink coverage in the $-100 \ldots 0$ window (ROI) upstream of the 3' splice site: 1) relative crosslink coverage iiclip_d1 > iiclip_nd1, 2) relative crosslink coverage iiclip_d1 < iiclip_nd1, 3) no crosslink detected in window. Relative crosslink coverage was calculated for each exon by counts of crosslinks in ROI divided by the sum of crosslinks in $\mathrm{ROI}$ across all silenced exons within the experiment. Motif coverage was visualised as a metaprofile and as a heatmap. In the heatmap, exons were ranked based on the total number of nucleotides within the ROI covered by any PTBP1 motifs to aid visualisation of the distribution of motifs.

\section{CLIP visualisation}

CLIP data were visualised in a comparative manner on individual transcripts or known PTBP1 regulated exons using the software clipplotr (https://github.com/ulelab/clipplotr), using the gencode v27 hg38 annotation. Library size normalisation or maxpeak normalisation were performed within clipplotr as indicated in figure legends. 


\section{References}

Agostini, Federico, Julian Zagalak, Jan Attig, Jernej Ule, and Nicholas M. Luscombe. 2021.

"Intergenic RNA Mainly Derives from Nascent Transcripts of Known Genes." Genome Biology 22 (1): 136.

Attig, Jan, Federico Agostini, Clare Gooding, Anob M. Chakrabarti, Aarti Singh, Nejc Haberman, Julian A. Zagalak, et al. 2018. "Heteromeric RNP Assembly at LINEs Controls Lineage-Specific RNA Processing." Cell 174 (5): 1067-81.e17.

Bagnoli, Johannes W., Christoph Ziegenhain, Aleksandar Janjic, Lucas E. Wange, Beate Vieth, Swati Parekh, Johanna Geuder, Ines Hellmann, and Wolfgang Enard. 2018. "Sensitive and Powerful Single-Cell RNA Sequencing Using mcSCRB-Seq.” Nature Communications 9 (1): 2937.

Blondal, Thorarinn, Audur Thorisdottir, Unnur Unnsteinsdottir, Sigridur Hjorleifsdottir, Arnthor Ævvarsson, Sveinn Ernstsson, Olafur H. Fridjonsson, et al. 2005. "Isolation and Characterization of a Thermostable RNA Ligase 1 from a Thermus Scotoductus Bacteriophage TS2126 with Good Single-Stranded DNA Ligation Properties." Nucleic Acids Research 33 (1): 135-42.

Brannan, Kristopher W., Isaac A. Chaim, Ryan J. Marina, Brian A. Yee, Eric R. Kofman, Daniel A. Lorenz, Pratibha Jagannatha, et al. 2021. "Robust Single-Cell Discovery of RNA Targets of RNA-Binding Proteins and Ribosomes." Nature Methods 18 (5): 507-19.

Buchbender, Andreas, Holger Mutter, F. X. Reymond Sutandy, Nadine Körtel, Heike Hänel, Anke Busch, Stefanie Ebersberger, and Julian König. 2019. "Improved Library Preparation with the New iCLIP2 Protocol." Methods , October. https://doi.org/10.1016/j.ymeth.2019.10.003.

Cereda, Matteo, Uberto Pozzoli, Gregor Rot, Peter Juvan, Anthony Schweitzer, Tyson Clark, and Jernej Ule. 2014. "RNAmotifs: Prediction of Multivalent RNA Motifs That Control Alternative Splicing." Genome Biology 15 (1): R20.

Chakrabarti, Anob M., Nejc Haberman, Arne Praznik, Nicholas M. Luscombe, and Jernej Ule. 2018. "Data Science Issues in Studying Protein-RNA Interactions with CLIP Technologies." Annual Review of Biomedical Data Science 1 (1): 235-61.

Chou, M. Y., J. G. Underwood, J. Nikolic, M. H. Luu, and D. L. Black. 2000. "Multisite RNA Binding and Release of Polypyrimidine Tract Binding Protein during the Regulation of c-Src NeuralSpecific Splicing." Molecular Cell 5 (6): 949-57.

Gueroussov, Serge, Thomas Gonatopoulos-Pournatzis, Manuel Irimia, Bushra Raj, Zhen-Yuan Lin, Anne-Claude Gingras, and Benjamin J. Blencowe. 2015. "An Alternative Splicing Event Amplifies Evolutionary Differences between Vertebrates." Science 349 (6250): 868-73.

Gu, Jiaqi, Ming Wang, Yang Yang, Ding Qiu, Yiqun Zhang, Jinbiao Ma, Yu Zhou, Gregory J. Hannon, and Yang Yu. 2018. "GoldCLIP: Gel-Omitted Ligation-Dependent CLIP." Genomics, Proteomics \& Bioinformatics 16 (2): 136-43.

Haberman, Nejc, Ina Huppertz, Jan Attig, Julian König, Zhen Wang, Christian Hauer, Matthias W. Hentze, et al. 2017. "Insights into the Design and Interpretation of iCLIP Experiments." Genome Biology 18 (1): 7.

Hafner, Markus, Maria Katsantoni, Tino Köster, James Marks, Joyita Mukherjee, Dorothee Staiger, Jernej Ule, and Mihaela Zavolan. 2021. "CLIP and Complementary Methods." Nature Reviews Methods Primers 1 (1): 1-23.

Hallegger, Martina, Anob M. Chakrabarti, Flora C. Y. Lee, Bo Lim Lee, Aram G. Amalietti, Hana M. Odeh, Katie E. Copley, et al. 2021. "TDP-43 Condensation Properties Specify Its RNA-Binding and Regulatory Repertoire." Cell, August. https://doi.org/10.1016/j.cell.2021.07.018.

Huppertz, Ina, Jan Attig, Andrea D'Ambrogio, Laura E. Easton, Christopher R. Sibley, Yoichiro Sugimoto, Mojca Tajnik, Julian König, and Jernej Ule. 2014. "iCLIP: Protein-RNA Interactions at Nucleotide Resolution." Methods 65 (3): 274-87.

König, Julian, Kathi Zarnack, Gregor Rot, Tomaz Curk, Melis Kayikci, Blaz Zupan, Daniel J. Turner, Nicholas M. Luscombe, and Jernej Ule. 2010. "iCLIP Reveals the Function of hnRNP Particles in Splicing at Individual Nucleotide Resolution." Nature Structural \& Molecular Biology 17 (7): 90915. 
Lama, Lodoe, Jose Cobo, Diego Buenaventura, and Kevin Ryan. 2019. "Small RNA-Seq: The RNA 5'-End Adapter Ligation Problem and How to Circumvent It." Journal of Biological Methods 6 (1). https://doi.org/10.14440/jbm.2019.269.

Lapointe, Christopher P., Daniel Wilinski, Harriet A. J. Saunders, and Marvin Wickens. 2015. "ProteinRNA Networks Revealed through Covalent RNA Marks." Nature Methods 12 (12): 1163-70.

Lee, Flora C. Y., and Jernej Ule. 2018. "Advances in CLIP Technologies for Studies of Protein-RNA Interactions." Molecular Cell 69 (3): 354-69.

Llorian, Miriam, Schraga Schwartz, Tyson A. Clark, Dror Hollander, Lit-Yeen Tan, Rachel Spellman, Adele Gordon, et al. 2010. "Position-Dependent Alternative Splicing Activity Revealed by Global Profiling of Alternative Splicing Events Regulated by PTB." Nature Structural \& Molecular Biology 17 (9): 1114-23.

McMahon, Aoife C., Reazur Rahman, Hua Jin, James L. Shen, Allegra Fieldsend, Weifei Luo, and Michael Rosbash. 2016. "TRIBE: Hijacking an RNA-Editing Enzyme to Identify Cell-Specific Targets of RNA-Binding Proteins." Cell 165 (3): 742-53.

Moggridge, Sophie, Poul H. Sorensen, Gregg B. Morin, and Christopher S. Hughes. 2018. "Extending the Compatibility of the SP3 Paramagnetic Bead Processing Approach for Proteomics." Journal of Proteome Research 17 (4): 1730-40.

Oberstrass, Florian C., Sigrid D. Auweter, Michèle Erat, Yann Hargous, Anke Henning, Philipp Wenter, Luc Reymond, et al. 2005. "Structure of PTB Bound to RNA: Specific Binding and Implications for Splicing Regulation." Science 309 (5743): 2054-57.

Pandya-Jones, Amy, Yolanda Markaki, Jacques Serizay, Tsotne Chitiashvili, Walter R. Mancia Leon, Andrey Damianov, Constantinos Chronis, et al. 2020. "A Protein Assembly Mediates Xist Localization and Gene Silencing." Nature 587 (7832): 145-51.

Quinlan, Aaron R., and Ira M. Hall. 2010. "BEDTools: A Flexible Suite of Utilities for Comparing Genomic Features." Bioinformatics. https://doi.org/10.1093/bioinformatics/btq033.

Ramanathan, Muthukumar, Douglas F. Porter, and Paul A. Khavari. 2019. "Methods to Study RNAProtein Interactions." Nature Methods 16 (3): 225-34.

Shen, Shihao, Juw Won Park, Zhi-Xiang Lu, Lan Lin, Michael D. Henry, Ying Nian Wu, Qing Zhou, and Yi Xing. 2014. "rMATS: Robust and Flexible Detection of Differential Alternative Splicing from Replicate RNA-Seq Data." Proceedings of the National Academy of Sciences of the United States of America 111 (51): E5593-5601.

Spellman, R., A. Rideau, A. Matlin, C. Gooding, F. Robinson, N. McGlincy, S. N. Grellscheid, J. Southby, M. Wollerton, and C. W. J. Smith. 2005. "Regulation of Alternative Splicing by PTB and Associated Factors." Biochemical Society Transactions 33 (Pt 3): 457-60.

Ule, Jernej, Kirk B. Jensen, Matteo Ruggiu, Aldo Mele, Aljaz Ule, and Robert B. Darnell. 2003. "CLIP Identifies Nova-Regulated RNA Networks in the Brain." Science 302 (5648): 1212-15.

Ule, Jernej, Kirk Jensen, Aldo Mele, and Robert B. Darnell. 2005. "CLIP: A Method for Identifying Protein-RNA Interaction Sites in Living Cells." Methods 37 (4): 376-86.

Ule, Jernej, Giovanni Stefani, Aldo Mele, Matteo Ruggiu, Xuning Wang, Bahar Taneri, Terry Gaasterland, Benjamin J. Blencowe, and Robert B. Darnell. 2006. "An RNA Map Predicting Nova-Dependent Splicing Regulation.” Nature 444 (7119): 580-86.

Van Nostrand, Eric L., Peter Freese, Gabriel A. Pratt, Xiaofeng Wang, Xintao Wei, Rui Xiao, Steven M. Blue, et al. 2020. "A Large-Scale Binding and Functional Map of Human RNA-Binding Proteins." Nature 583 (7818): 711-19.

Van Nostrand, Eric L., Gabriel A. Pratt, Alexander A. Shishkin, Chelsea Gelboin-Burkhart, Mark Y. Fang, Balaji Sundararaman, Steven M. Blue, et al. 2016. "Robust Transcriptome-Wide Discovery of RNA-Binding Protein Binding Sites with Enhanced CLIP (eCLIP)." Nature Methods 13 (6): 508-14.

Witten, Joshua T., and Jernej Ule. 2011. "Understanding Splicing Regulation through RNA Splicing Maps." Trends in Genetics: TIG 27 (3): 89-97.

Xue, Yuanchao, Yu Zhou, Tongbin Wu, Tuo Zhu, Xiong Ji, Young-Soo Kwon, Chao Zhang, et al. 2009. "Genome-Wide Analysis of PTB-RNA Interactions Reveals a Strategy Used by the 
bioRxiv preprint doi: https://doi.org/10.1101/2021.08.27.457890; this version posted August 27, 2021. The copyright holder for this preprint

(which was not certified by peer review) is the author/funder, who has granted bioRxiv a license to display the preprint in perpetuity. It is made available under aCC-BY-NC-ND 4.0 International license.

General Splicing Repressor to Modulate Exon Inclusion or Skipping." Molecular Cell 36 (6): 9961006.

Zarnegar, Brian J., Ryan A. Flynn, Ying Shen, Brian T. Do, Howard Y. Chang, and Paul A. Khavari. 2016. "irCLIP Platform for Efficient Characterization of protein-RNA Interactions." Nature Methods 13 (6): 489-92. 
Day $1 / 2$

Crosslinking to

Ligation

1

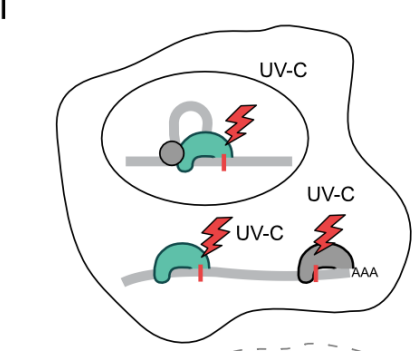

2

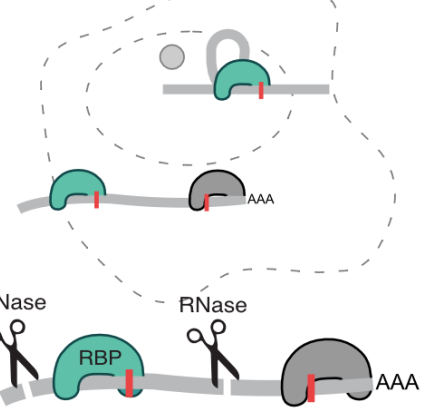

4

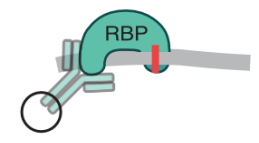

5

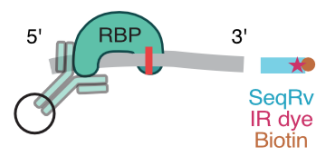

Day 2/3

Elution from beads

to RNA extraction

$6_{\text {RNase }}$

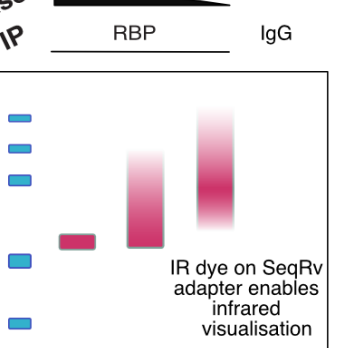

7
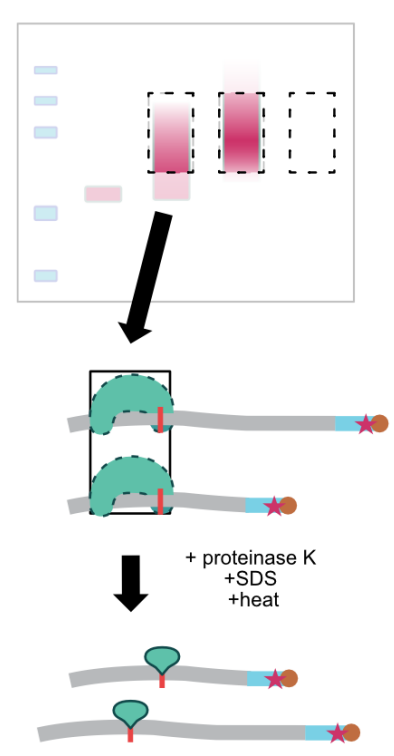

Day 3/4

Reverse transcription

to PCR amplification

8

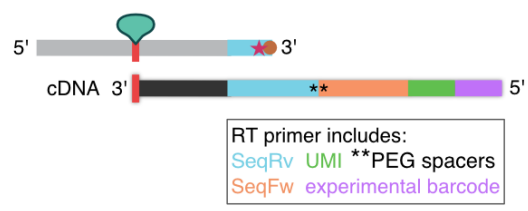

9

beads-based cleanup of cDNAs

10

intramolecular ligation

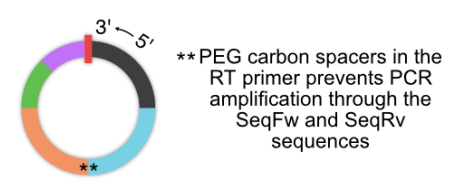

11

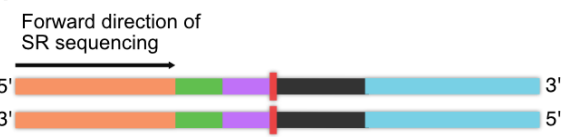

Optional gel purification after PCR
amplification

Figure 1: iiCLIP overview

Schematic of iiCLIP. There are 11 main experimental stages in CLIP and variant protocols, as previously defined in Lee and Ule (2018). These include: 1. Covalent protein-RNA crosslinking 2. Cell lysis 3. RNA fragmentation 4. Purification of protein-RNA complexes 5. Ligation of SeqRv adapter to fragmented RNA 6. Quality control by visualisation of captured protein-RNA complexes 7. RNA extraction 8. Reverse transcription 9. cDNA purification 10. SeqFw adapter ligation 11. cDNA amplification followed by high-throughput sequencing of multiplexed libraries. Stages where key changes have been introduced are described in Table 1. The protocol can be completed from UV crosslinking to library preparation in 3/4 days. 


\section{a}

Batched
Input

UVC crosslinking

1 million cells

RNase I

PTBP1 Antibody
2 Protocols Performed in 3 Labs
iCLIP2 in

developer lab

iiCLIP in non-developer lab

b
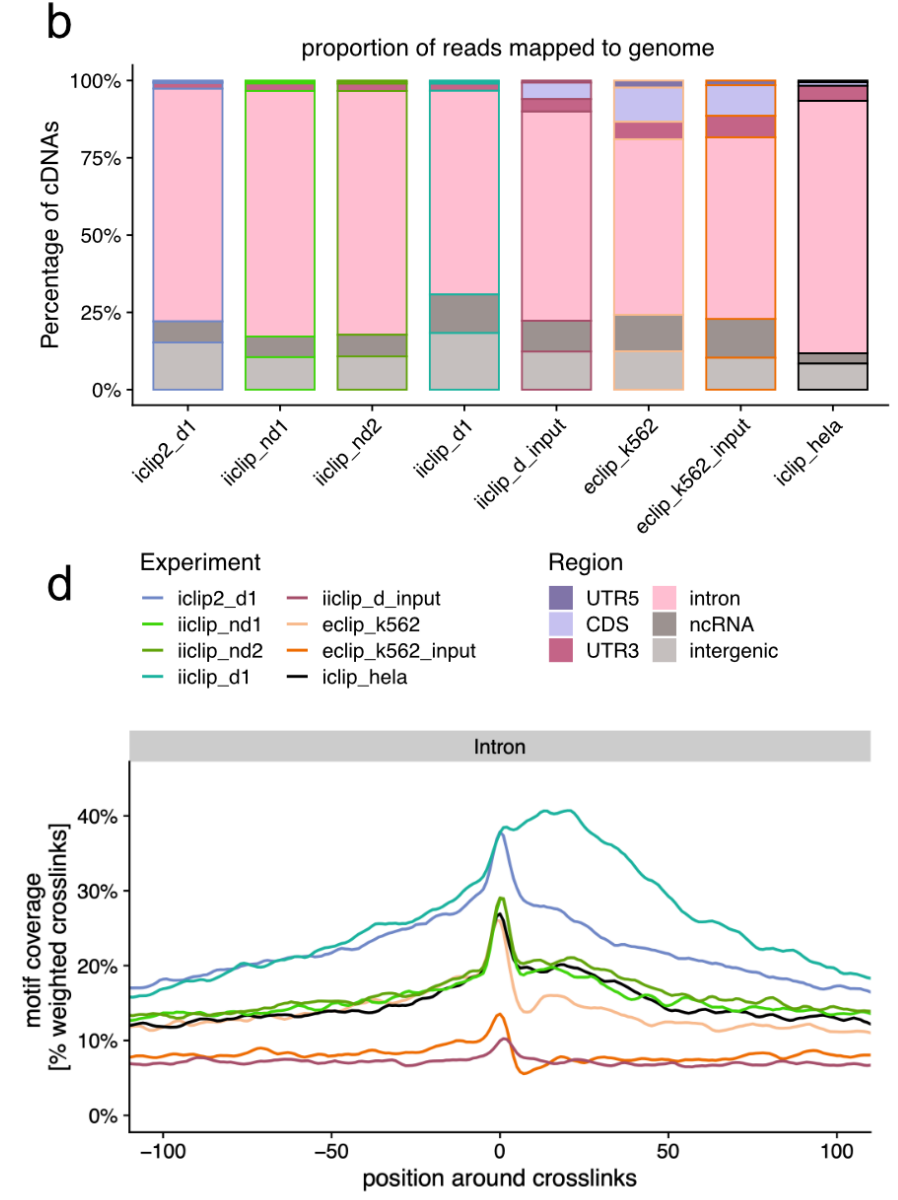

Benchmarking of iiCLIP and validation with public eCLIP and iCLIP
Reproducible assignment of crosslinks from sequenced data using truncationbased approach
Motif enrichment

RNAmaps analysis

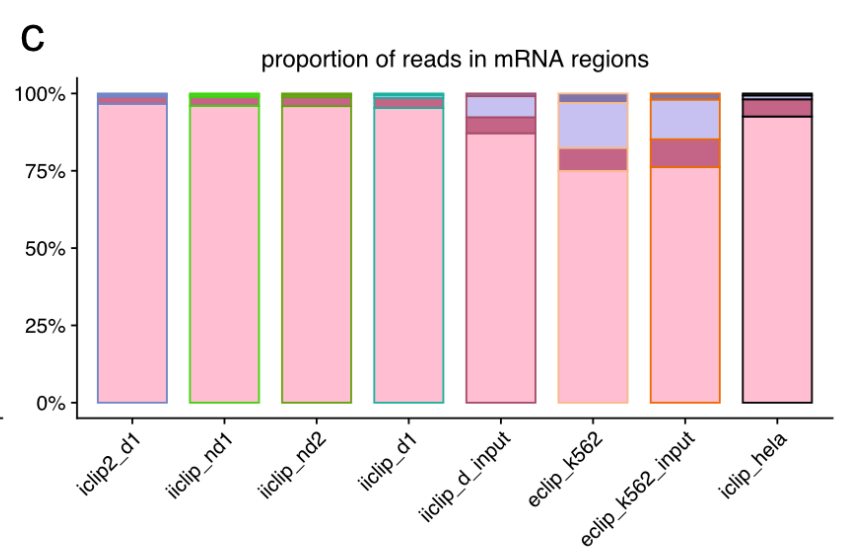

e

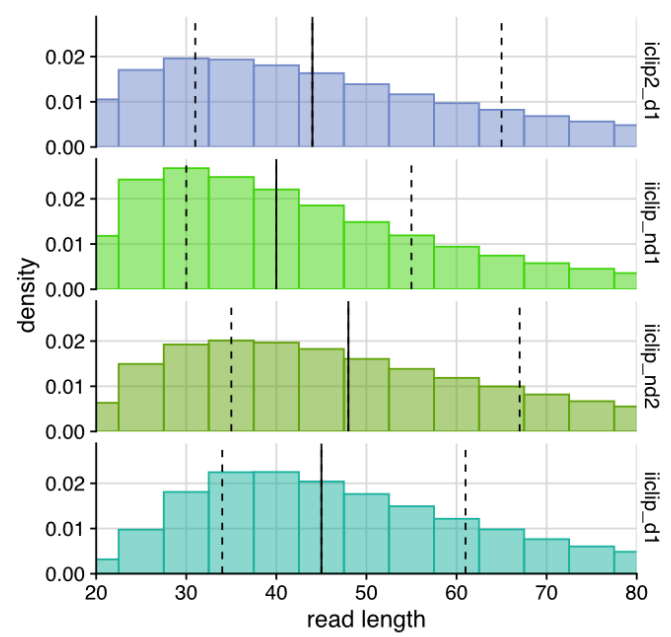

Figure 2: Regional and motif enrichment in identified raw PTBP1 crosslink positions

a) Design of iiCLIP benchmarking comparisons. Input material is controlled appropriately between iiCLIP and iCLIP2 libraries, and downstream bioinformatic analysis is done systematically, including for public eCLIP and iCLIP data. (b-c) Regional distribution of cDNAs mapped to (b) genome or (c) filtered for mRNA regions (CDS, 5'UTR, 3'UTR, intron) identified by the different protocols and experiments. d) Enrichment of known PTBP1 pentamers around crosslink positions (\% crosslinks weighted by cDNA counts with motif coverage) in intronic regions. (e) Histogram showing read length distribution of uniquely genome-mapping reads. Solid line indicates the median read length, and dashed lines indicate the lower and upper quartiles. 

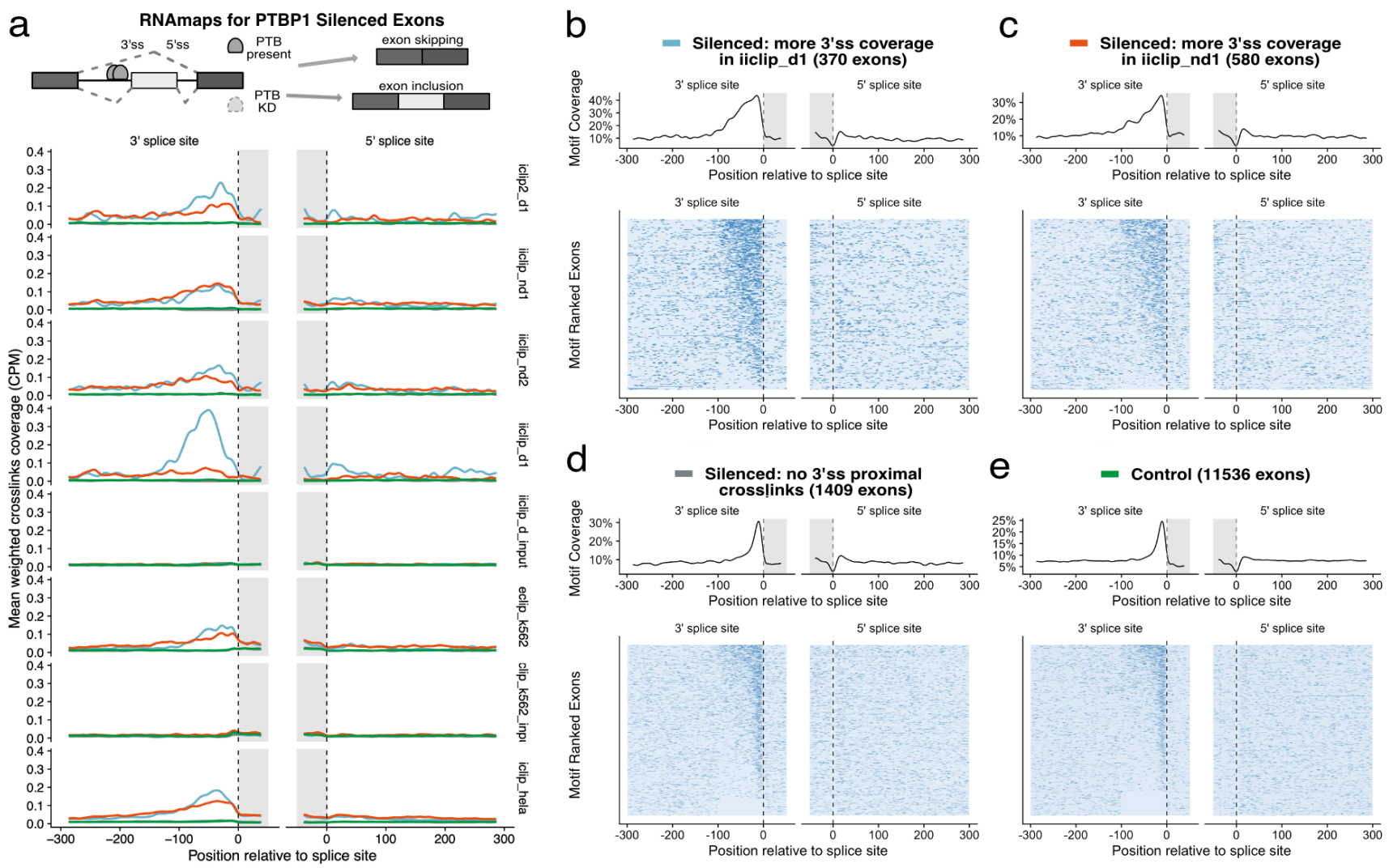

motif $0 \square 1$

Figure 3: PTBP1 RNA maps evaluate the sensitivity and specificity across methods

a) In the following PTBP1 RNA maps, weighted crosslinks normalised by library size are plotted as a metaprofile around the 3' and 5' splice sites of PTBP1 silenced compared with control (Green) exons identified from public PTBP1/2 KD RNAseq. Silenced exons were stratified into 3 classes based on crosslink site coverage proximal to the 3'ss (-100..0 window). Light blue: more covered in iiclip_d1 compared to iiclip_nd1; Orange: more covered in iiclip_nd1 compared to iiclip_d1; Grey: no detected crosslinks from both libraries. The profile is used to evaluate the specificity and sensitivity of each method.

b-e) Motif maps for each of the 3 classes of PTBP1 silenced exons (b-d) and the control exons (e), shown as a coverage metaprofile (top) or heatmap (bottom). (b) More covered in iiclip_d1 compared to iiclip_nd1; (c) more covered in iiclip_nd1 compared to iiclip_d1; (d) no detected crosslinks from both libraries. 
a $\quad$ PTBP1 CLIP

iCLIP2 dev rep1 $\quad$ iiCLIP nondev rep1 iiCLIP nondev rep2 $\quad$ iiCLIP dev rep1

ENCODE RNA-seq

- - K562 control rep1 = - K562 control rep2 = - K562 PTBP1 CRISPR rep1 = - K562 PTBP1 CRISPR rep2

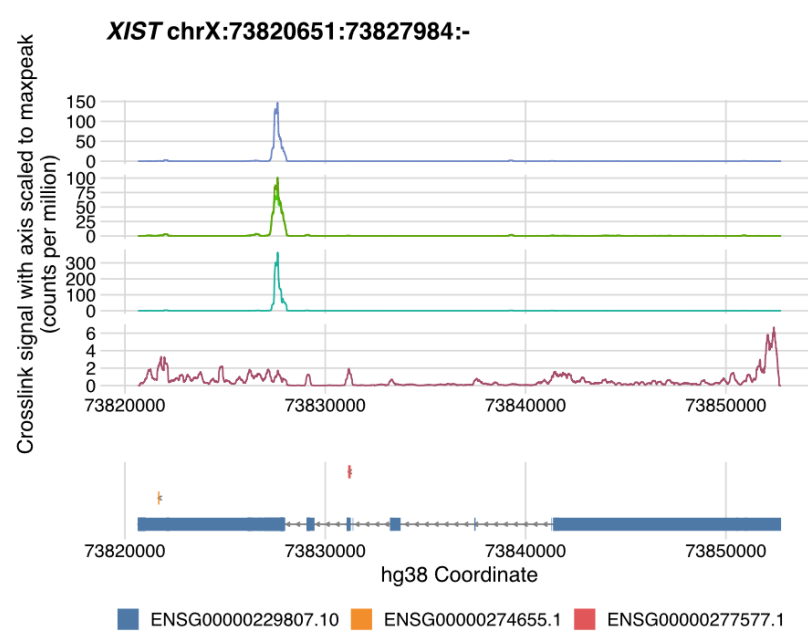

MALAT1 chr11:65497591:65507759:+

b

PTBP2 exon 10 chr1:96806219:96806652:+
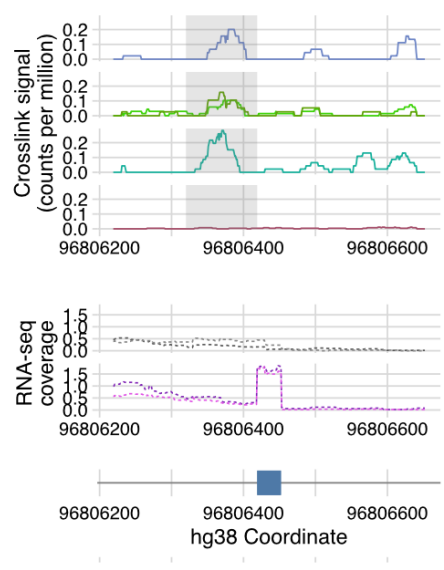

ENSG00000117569.18
DPF2 exon 7 chr11:65344250:65345000:+
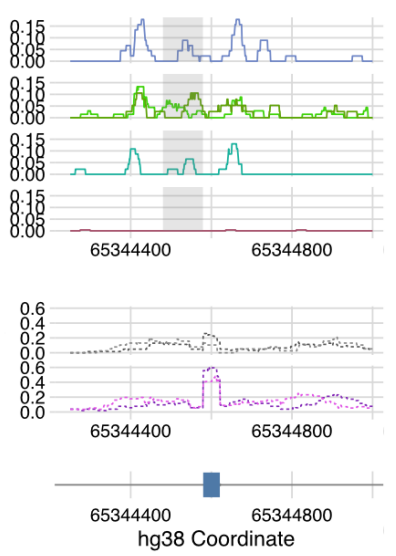

ENSG00000133884.9
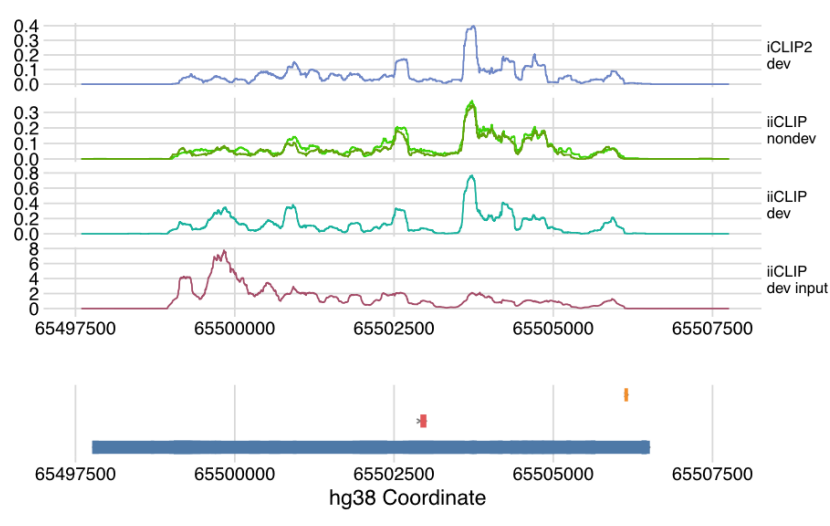

ENSG00000251562.7 [ ENSG00000274072.1 — ENSG00000278217.1

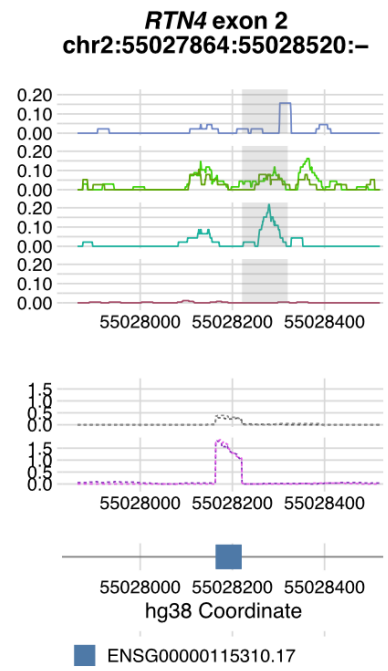

( PTBP3 exon 2
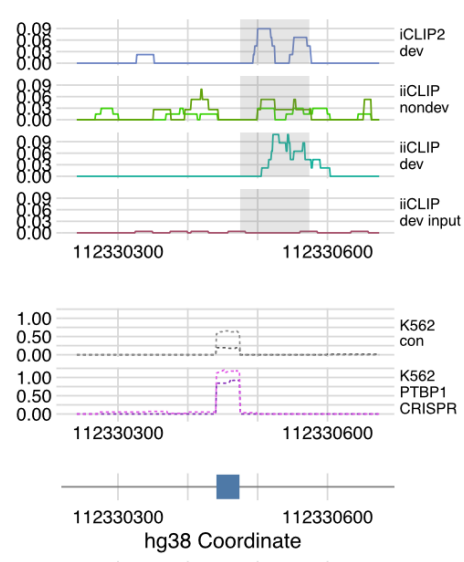

ENSG00000119314.15

Figure 4: Visualisation of PTBP1 iCLIP2 and iiCLIP crosslink events on PTBP1 RNA binding sites

(a) Comparative visualisation of PTBP1 CLIP libraries on ncRNA targets XIST and MALAT1. Top panel: library size normalised crosslink counts from PTBP1 CLIP libraries generated for this study are plotted. Axis is scaled by the maximum peak height of each group to visualise the distribution of crosslink signal in the input library, containing global protein-RNA crosslinking signal, compared to PTBP1 crosslink signal. Bottom panel: Gene annotation (GENCODE hg38 v27). (b) Comparative visualisation of PTBP1 CLIP libraries on individual intronic sites surrounding regulated exons. Top panel: library size normalised crosslinking counts from PTBP1 CLIP libraries generated for this study are plotted, centred on examples of previously validated PTBP1 repressed cassette exons. The region of $-100 \ldots 0$ upstream of the 3' splice site is shaded in grey, as this region contains the highest reproducible enrichment of crosslinks across silenced exons as shown previously in the RNAmaps metaprofiles. Middle panel: RNAseq coverage (CPM) from ENCODE K562 control and PTBP1 CRISPR libraries. Cassette exon inclusion increases upon PTBP1 CRISPR treatment. Bottom panel: Gene annotation (GENCODE hg38 v27). 

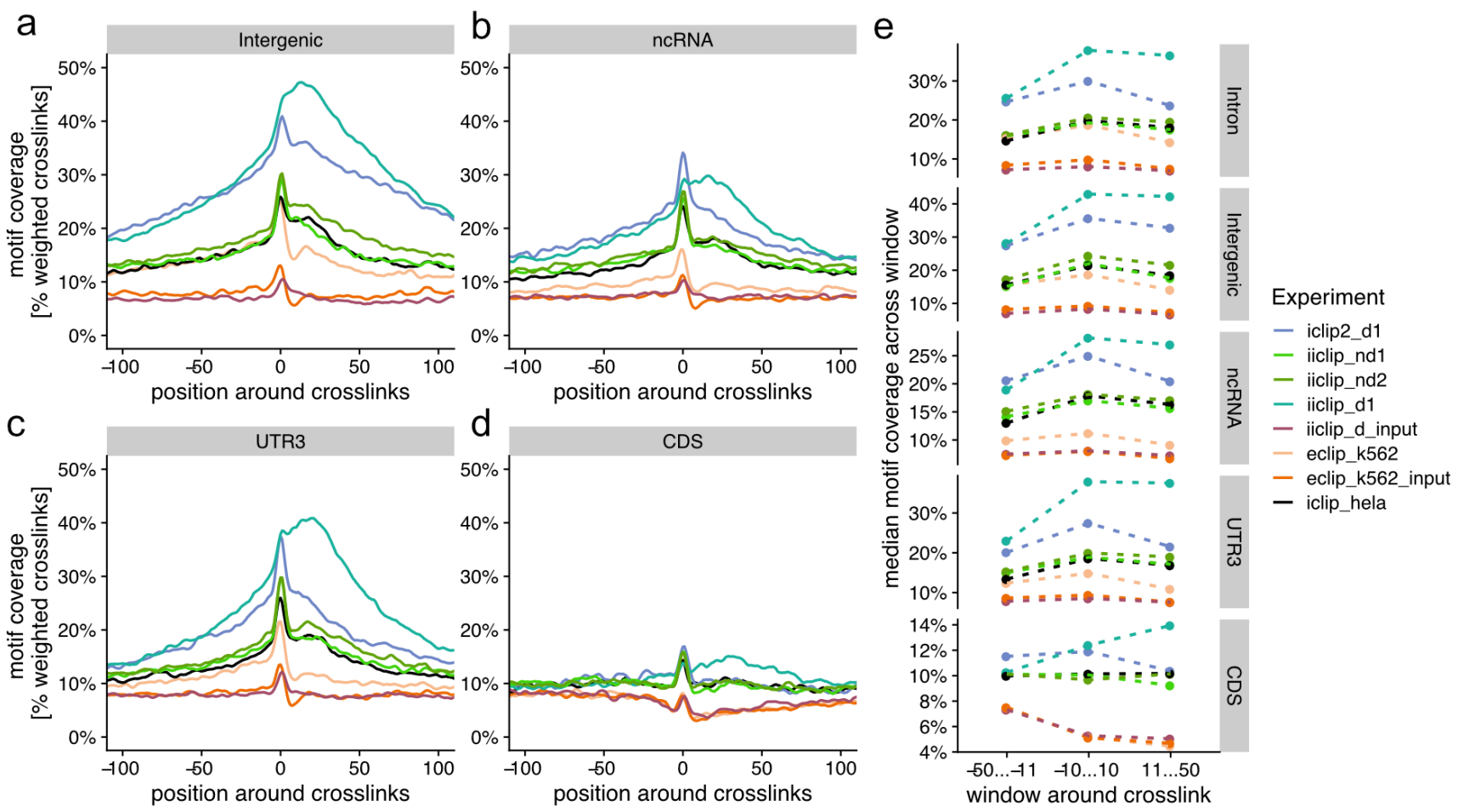

Figure S1: Motif enrichment in PTBP1 and Input crosslink positions across Intergenic, ncRNA, 3' UTR and CDS regions.

Enrichment of known PTBP1 pentamers around weighted crosslink positions in (a) intergenic, (b) ncRNA, (c) 3' UTR and (d) CDS regions. (e) To summarise the proximal as well as broader enrichment of motif, showing the median motif enrichment of each method in 3 windows upstream, centred or downstream relative to crosslink position. 


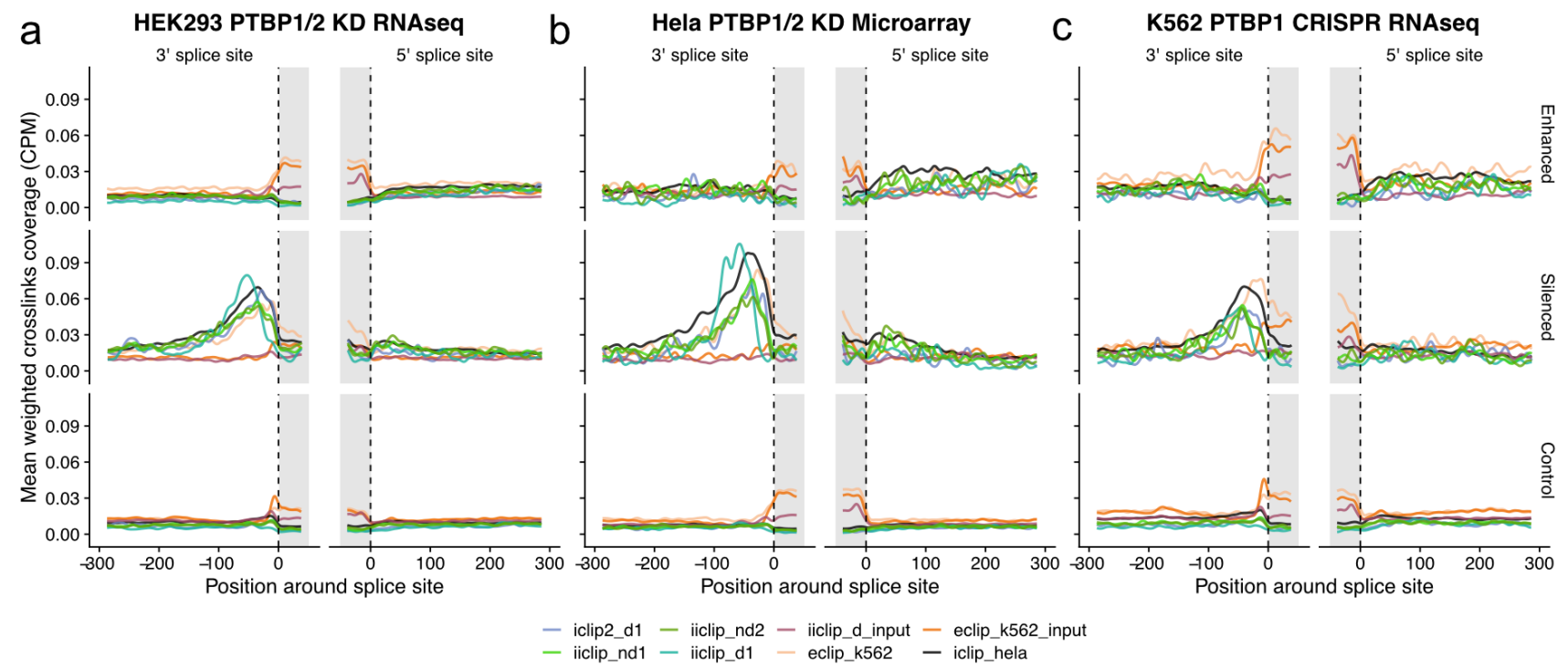

Figure S2: PTBP1 RNA maps evaluate the sensitivity and specificity across methods

PTBP1 RNA maps summarised for enhanced (top), silenced (middle) and control (bottom) exons from 3 public PTB KD datasets: (a) HEK293 PTBP1/2 KD RNAseq analysis (related to Fig.3), (b) HELA PTBP1/2 KD microarray data and (c) K562 PTBP1 CRISPR-treated RNAseq analysis. 
Table 1: summary and rationale of optimisations

\begin{tabular}{|c|c|c|}
\hline $\begin{array}{l}\text { Previous iCLIP (Huppertz et } \\
\text { al. 2014) }\end{array}$ & iiCLIP & Rationale \\
\hline \multirow[t]{2}{*}{ 5. Ligation of L3 adapter } & $\begin{array}{l}\text { Ligation reaction contains } \\
\text { DMSO and no DTT, as in } \\
\text { eCLIP (Van Nostrand et al. } \\
\text { 2016) }\end{array}$ & May improve ligation efficiency \\
\hline & $\begin{array}{l}\text { Enzymatic removal of adapter } \\
\text { after ligation }\end{array}$ & $\begin{array}{l}\text { Improves the efficiency of } \\
\text { downstream steps by } \\
\text { minimising artefacts that can be } \\
\text { caused by adapter carry-over }\end{array}$ \\
\hline 6. Radioactive imaging & $\begin{array}{l}\text { Infrared imaging enabled by } \\
\text { ligation of infrared adapter as in } \\
\text { irCLIP (Zarnegar et al. 2016) }\end{array}$ & $\begin{array}{l}\text { Convenience, health and safety } \\
\text { precautions }\end{array}$ \\
\hline \multirow[t]{2}{*}{$\begin{array}{l}\text { 7. Proteinase } \mathrm{K}(\mathrm{PK}) \text { digestion } \\
\text { in Urea-containing buffer }\end{array}$} & $\begin{array}{l}\text { PK digestion in SDS-containing } \\
\text { buffer (Zarnegar et al. 2016) }\end{array}$ & $\begin{array}{l}\text { Convenience. Both urea and } \\
\text { SDS enhance PK activity. } \\
\text { Unlike the PK-urea buffer, PK- } \\
\text { SDS buffer does not need to be } \\
\text { made fresh every time as it is } \\
\text { more stable. }\end{array}$ \\
\hline & $\begin{array}{l}\text { Addition of reverse transcription } \\
\text { primer into ethanol precipitation }\end{array}$ & $\begin{array}{l}\text { Increased reliability of ethanol } \\
\text { precipitation by minimising loss } \\
\text { of material }\end{array}$ \\
\hline \multirow[t]{2}{*}{$\begin{array}{l}\text { 8. Reverse transcription with } \\
\text { SSIII }\end{array}$} & Reverse transcription with SSIV & $\begin{array}{l}\text { Shorter time of reverse } \\
\text { transcription, increased } \\
\text { efficiency }\end{array}$ \\
\hline & $\begin{array}{l}\text { Addition of PEG8000 into } \\
\text { reverse transcription }\end{array}$ & $\begin{array}{l}\text { Increased reverse transcription } \\
\text { efficiency (Bagnoli et al. 2018) }\end{array}$ \\
\hline $\begin{array}{l}\text { 9. Gel-based size selection of } \\
\text { cDNA before circularisation }\end{array}$ & $\begin{array}{l}\text { Isopropanol ampure beads- } \\
\text { based purification of cDNAs }\end{array}$ & $\begin{array}{l}\text { Convenience and increased } \\
\text { yield. }\end{array}$ \\
\hline
\end{tabular}




\begin{tabular}{|c|c|c|}
\hline & before circularisation & \\
\hline \multirow[t]{2}{*}{$\begin{array}{l}\text { 10. Circularisation with } \\
\text { circligase II for cDNA adapter } \\
\text { ligation }\end{array}$} & $\begin{array}{l}\text { Option 1) Circularisation with } \\
\text { commercial circligase II with } \\
\text { betaine additive }\end{array}$ & $\begin{array}{l}\text { Betaine increases the efficiency } \\
\text { of circularisation reaction when } \\
\text { used with circligase II }\end{array}$ \\
\hline & $\begin{array}{l}\text { Option 2) Circularisation with } \\
\text { in-house purified TS2126 ligase } \\
\text { (Blondal et al. 2005) } \\
\text { (equivalent to commercial } \\
\text { circligase I) }\end{array}$ & $\begin{array}{l}\text { In-house produced ligase has } \\
\text { high circularisation efficiency } \\
\text { (Lama et al. 2019) and greatly } \\
\text { decreased the cost for large- } \\
\text { scale experiments. }\end{array}$ \\
\hline $\begin{array}{l}\text { 10. Circularisation followed by } \\
\text { BamHI linearisation }\end{array}$ & $\begin{array}{l}\text { Circularisation with RT primers } \\
\text { that contain carbon spacers as } \\
\text { in irCLIP (Zarnegar et al. 2016) }\end{array}$ & $\begin{array}{l}\text { Carbon spacers within the RT } \\
\text { primers stop the progression of } \\
\text { PCR, removing the need for } \\
\text { linearisation }\end{array}$ \\
\hline
\end{tabular}


Table S1: Information on PTB benchmarking experiments

\begin{tabular}{|l|l|l|l|l|l|}
\hline Label & $\begin{array}{l}\text { Particip } \\
\text { ating lab }\end{array}$ & Protocol & Replicates & $\begin{array}{l}\text { Sequencin } \\
\mathbf{g}\end{array}$ & Comments \\
\hline $\begin{array}{l}\text { iCLIP2 in } \\
\text { developer lab } \\
\text { (iCLIP2_d) }\end{array}$ & Konig & iCLIP2 & 1 & $\begin{array}{l}\text { SR150, } \\
\text { Miseq. }\end{array}$ & $\begin{array}{l}\text { For consistency with other } \\
\text { libraries, reads were } \\
\text { preprocessed to be } \\
\text { truncated to 100 read } \\
\text { length }\end{array}$ \\
\hline $\begin{array}{l}\text { iiCLIP in non- } \\
\text { developer lab } \\
\text { (iiCLIP_nd) }\end{array}$ & Turner & iiCLIP & 2 & $\begin{array}{l}\text { SR100, } \\
\text { Hiseq4000. }\end{array}$ & $\begin{array}{l}\text { without RT primer in } \\
\text { ethanol precipitation, RT } \\
\text { without PEG8000 and } \\
\text { circularisation using } \\
\text { commercial circligase II }\end{array}$ \\
\hline $\begin{array}{l}\text { iiCLIP in } \\
\text { developer lab } \\
\text { (iiCLIP_d) }\end{array}$ & Ule & iiCLIP & 1 & $\begin{array}{l}\text { Hiseq4000. } \\
\text { precipitation, RT with } \\
\text { PEG8000 and } \\
\text { circularisation using in- } \\
\text { house TS2126 RNA ligase }\end{array}$ \\
\end{tabular}


bioRxiv preprint doi: https://doi.org/10.1101/2021.08.27.457890; this version posted August 27, 2021 . The copyright holder for this preprint (which was not certified by peer review) is the author/funder, who has granted bioRxiv a license to display the preprint in perpetuity. It is made available under aCC-BY-NC-ND 4.0 International license.

Table S2: PTBP1 library statistics across community and public datasets

\begin{tabular}{|c|c|c|c|c|c|c|c|}
\hline experiment & $\begin{array}{l}\text { tRNA/rRN } \\
\text { A } \\
\text { uniquely } \\
\text { mapped } \\
\text { reads } \\
\text { (Multimap } \\
\text { ping } \\
\text { reads) }\end{array}$ & $\begin{array}{l}\text { Genome } \\
\text { uniquely } \\
\text { mapped } \\
\text { reads } \\
\text { (Multimappi } \\
\text { ng reads) }\end{array}$ & $\begin{array}{l}\text { tRNA/r } \\
\text { RNA : } \\
\text { Geno } \\
\text { me } \\
\text { ratio }\end{array}$ & $\begin{array}{l}\text { Genome } \\
\text { uniquely } \\
\text { mapped } \\
\text { cDNAs } \\
\text { after UMI } \\
\text { deduplicati } \\
\text { on }\end{array}$ & $\begin{array}{l}\text { PCR } \\
\text { duplic } \\
\text { ation } \\
\text { ratio }\end{array}$ & $\begin{array}{l}\text { Raw } \\
\text { crosslink } \\
\text { positions }\end{array}$ & $\begin{array}{l}\text { cDNAs : } \\
\text { position } \\
\text { ratio }\end{array}$ \\
\hline iCLIP2_d1 & $\begin{array}{l}50,211 \\
(11,590)\end{array}$ & $\begin{array}{l}2,654,186 \\
(603,861)\end{array}$ & 0.0190 & $1,791,751$ & 1.48 & $1,197,155$ & 1.49 \\
\hline iiCLIP_nd1 & $\begin{array}{l}88,399 \\
(16,956)\end{array}$ & $\begin{array}{l}5,791,534 \\
(710,378)\end{array}$ & 0.0162 & $2,712,629$ & 2.14 & $2,362,677$ & 1.15 \\
\hline iiCLIP_nd2 & $\begin{array}{l}66,758 \\
(10,200)\end{array}$ & $\begin{array}{l}6,947,194 \\
(638,421)\end{array}$ & 0.0101 & $1,515,527$ & 4.58 & 1,327, 756 & 1.14 \\
\hline iiCLIP_d1 & $\begin{array}{l}184,969 \\
(16,591)\end{array}$ & $\begin{array}{l}5,813,447 \\
(1,912,303)\end{array}$ & 0.0261 & $1,844,251$ & 3.15 & $1,257,376$ & 1.47 \\
\hline $\begin{array}{l}\text { iiCLIP_ } \\
\text { d_Input }\end{array}$ & $\begin{array}{l}4,124,480 \\
(285,320)\end{array}$ & $\begin{array}{l}12,545,013 \\
(2,847,966)\end{array}$ & 0.286 & $10,420,964$ & 1.20 & $9,019,011$ & 1.16 \\
\hline $\begin{array}{l}\text { public_K562 } \\
\text { eCLIP_rep1 }\end{array}$ & $\begin{array}{l}1,458,797 \\
(4,438)\end{array}$ & $\begin{array}{l}2,612,129 \\
(518,084)\end{array}$ & 0.467 & $2,592,469$ & 1.01 & $2,171,418$ & 1.19 \\
\hline $\begin{array}{l}\text { public_K562 } \\
\text { eCLIP_rep2 }\end{array}$ & $\begin{array}{l}2,779,381 \\
(12,430)\end{array}$ & $\begin{array}{l}4,162,345 \\
(791,761)\end{array}$ & 0.564 & $4,116,381$ & 1.01 & $3,314,798$ & 1.24 \\
\hline $\begin{array}{l}\text { public_K562 } \\
\text { eCLIP_SMInp } \\
\text { ut }\end{array}$ & $\begin{array}{l}14,028,69 \\
5(35,264)\end{array}$ & $\begin{array}{l}5,976,586 \\
(1,153,157)\end{array}$ & 1.97 & $5,927,117$ & 1.01 & $4,997,591$ & 1.19 \\
\hline $\begin{array}{l}\text { public_HeLa_i } \\
\text { CLIP_rep1 }\end{array}$ & $\begin{array}{l}163,665 \\
(29,142)\end{array}$ & $\begin{array}{l}13,670,683 \\
(1,526,460)\end{array}$ & 0.0127 & $11,243,177$ & 1.22 & $8,886,744$ & 1.27 \\
\hline $\begin{array}{l}\text { public_HeLa_i } \\
\text { CLIP_rep2 }\end{array}$ & $\begin{array}{l}264,508 \\
(53,231)\end{array}$ & $\begin{array}{l}22,565,237 \\
(2,508,386)\end{array}$ & 0.0127 & $18,132,756$ & 1.24 & $13,403,391$ & 1.35 \\
\hline $\begin{array}{l}\text { public_HeLa_i } \\
\text { CLIP_rep3 }\end{array}$ & $\begin{array}{l}83,411 \\
(17,561)\end{array}$ & $\begin{array}{l}7,136,585 \\
(802,000)\end{array}$ & 0.0127 & $5,686,647$ & 1.25 & $4,831,520$ & 1.18 \\
\hline $\begin{array}{l}\text { public_HeLa_i } \\
\text { CLIP_rep4 }\end{array}$ & $\begin{array}{l}215,372 \\
(33,080)\end{array}$ & $\begin{array}{l}12,739,108 \\
(1,436,925)\end{array}$ & 0.0175 & $10,630,214$ & 1.20 & $8,534,781$ & 1.25 \\
\hline
\end{tabular}


Table S3: source of data

\begin{tabular}{|l|l|l|}
\hline experiment & Processed data & Publication \\
\hline iCLIP2_d1 & https://imaps.goodwright.com/samples/15590/ & This paper. \\
\hline iiCLIP_nd1 & https://imaps.goodwright.com/samples/15596/ & This paper. \\
\hline iiCLIP_nd2 & https://imaps.goodwright.com/samples/15595/ & This paper. \\
\hline iiCLIP_d1 & https://imaps.goodwright.com/samples/15581/ & This paper. \\
\hline iiCLIP_d_input & https://imaps.goodwright.com/samples/15582/ & This paper. \\
\hline $\begin{array}{l}\text { eCLIP_K562 (2 } \\
\text { replicates) }\end{array}$ & $\begin{array}{l}\text { https://imaps.goodwright.com/samples/15583/ } \\
\text { https://imaps.goodwright.com/samples/15584/ }\end{array}$ & $\begin{array}{l}\text { (Van Nostrand et } \\
\text { al. 2020) }\end{array}$ \\
\hline $\begin{array}{l}\text { eCLIP_K562_input } \\
\text { https://imaps.goodwright.com/samples/15585/ }\end{array}$ & $\begin{array}{l}\text { (Van Nostrand et } \\
\text { al. 2020) }\end{array}$ \\
\hline iCLIP_hela (4 \\
replicates) & $\begin{array}{l}\text { https://imaps.goodwright.com/samples/15586/ } \\
\text { https://imaps.goodwright.com/samples/15587/ } \\
\text { https://imaps.goodwright.com/samples/15588/ } \\
\text { https://imaps.goodwright.com/samples/15589/ }\end{array}$ & $\begin{array}{l}\text { (Attig et al. 2018; } \\
\text { Haberman et al. }\end{array}$ \\
\hline
\end{tabular}

